\title{
Resiliencia: competencia profesional del administrador de empresas
}

Resilience: professional competence of the business administrator

Boris Delgado Litardo

José Alejandro Flores Sánchez

Humberto Pedro Segarra Jaime

Johana Katiusca Meza Salvatierra

Fecha de recepción: 07 de noviembre de 2020

Fecha de aceptación: 21 de diciembre de 2020 


\title{
Resiliencia: competencia profesional del administrador de empresas
}

\author{
Resilience: professional competence of the business administrator \\ Boris Delgado Litardo ${ }^{1}$, José Alejandro Flores Sánchez ${ }^{2}$, Humberto Pedro Segarra Jaime ${ }^{3}$ y \\ Johana Katiusca Meza Salvatierra4
}

Como citar: Delgado, B., Flores, J., Segarra, H., Meza, J. (2021). Resiliencia: competencia profesional del administrador de empresa, Revista Universidad de Guayaquil. 132(1), 1-9. DOI: https://doi.org/10.53591/rug.v132i1.1350

\section{RESUMEN}

En la actualidad, ha tomado mayor relevancia identificar mecanismos de evaluación continua y procesos de negocio con el objetivo de absorber los impactos y dar una respuesta adecuada al cambio. La presente investigación tiene como objetivo realizar una revisión documental del término Resiliencia, su origen, características, beneficios y su aplicación en las diferentes disciplinas sociales y organizacionales. Mencionar como el comportamiento individual y colectivo del ser humano, puede afectar a la estabilidad empresarial. Además, explicar cómo esta habilidad es una competencia clave para administradores de empresas, a través de la implementación del pensamiento estratégico transformador, creando un ambiente laboral, más comunicativo y comprometido. Finalmente, se evidencia la importancia de realizar estudios complementario post pandemia del manejo de crisis y planes de continuidad empresarial.

PALABRAS CLAVE: Resiliencia organizacional, pensamiento estratégico, gestión de crisis

\begin{abstract}
At present, it has become more relevant to identify continuous evaluation mechanisms and business processes with the aim of absorbing impacts and giving an adequate response to change. The objective of this research is to carry out a documentary review of the term resilience, its origin, characteristics, benefits, and its application in the different social and organizational disciplines. Mention how the individual and collective behavior of the human being can affect business stability. Furthermore, to explaining how this ability is a key competence for business managers, through the implementation of transformative strategic thinking, creating a more communicative and committed work environment. Finally, the importance of carrying out complementary post-pandemic studies of crisis management and business continuity plans is evident.
\end{abstract}

KEYWORDS: Organizational resilience, strategic thinking, crisis management

\footnotetext{
${ }^{1}$ Master en Administración de Empresas. Docente de la Facultad de Ciencias Administrativas, Universidad de Guayaquil, Ecuador. Correo electrónico: boris.delgadol@ug.edu.ec. ORCID: https://orcid.org/0000-0003-4287-0744

${ }^{2}$ Magister en Derecho Constitucional. Docente de la Facultad de Ciencias Administrativa, Universidad de Guayaquil, Ecuador. Correo electrónico: jose.floressa@ug.edu.ec. ORCID: https://orcid.org/0000-0003-2246-5338

${ }^{3}$ Magister Ejecutivo en Dirección de Empresas con Énfasis en Gerencia Estratégica. Docente de la Facultad de Ciencias Administrativa, Universidad de Guayaquil, Ecuador. Correo electrónico: humberto.segarraj@ug.edu.ec, ORCID: https://orcid.org/0000-0003-3946-952X

${ }^{4}$ Magister en Administración de Empresas con Mención en Marketing. Docente de la Facultad de Ciencias Administrativa, Universidad de Guayaquil, Ecuador. Correo electrónico: johana.mezas@ug.edu.ec, ORCID: https://orcid.org/0000-0001-7671-5203
} 


\section{INTRODUCCIÓN}

En la actualidad, las condiciones económicas cambiantes, los requerimientos sociales derivados de la integración de diversas culturas y avances tecnológicos se producen de una forma muy acelerada. La era digital, la globalización y los clientes más exigentes han llevado a una evolución en el comportamiento del mercado y la dirección de las organizaciones, incorporando consideraciones adicionales de las políticas de precios, de la mano de un compromiso con la responsabilidad social (Revista Ekos Investigacion, 2011). Las transformaciones tecnológicas, demográficas, económicas deben hacer reflexionar a las organizaciones, que su objetivo sea el seguir en el camino del desarrollo sostenible, es decir, en la búsqueda de un cambio tanto gradual como direccional, no perpetuar una situación existente sino buscar un constante cambio (Gallopin, 2013).

Ante la crisis sanitaria mundial, durante el año 2020, las empresas fueron forzadas a operar en un entorno operativo incierto requiriendo la evaluación continua del riesgo, analizar la gestión de crisis y las estrategias para la continuidad del negocio. El distanciamiento social, confinamiento y restricciones de movilidad evidenciaron, e inclusive en algunos casos, profundizaron la mala calidad de vida, el estrés, los sacrificios familiares y personales. Varias medidas de contingencias fueron aplicadas para sobresalir, demandando la integración de personas y la tecnología, adoptar plataformas digitales, incrementar la ciberseguridad, mejorar la velocidad de la comunicación y calidad de información e innovar para garantizar la supervivencia de varios negocios.

La velocidad con que el mundo se mueve y en donde las empresas cada vez son menos rentables y algunas hasta se ven obligadas a liquidar. Es indispensable pensar seriamente en cómo contar con la capacidad para reinventarse y establecer estrategias resilientes para un modelo de negocio (Hamel \& Välikangas, 2003). Esta capacidad de resistir situaciones difíciles y de cultivar la misma, a lo largo del tiempo, es lo que se denomina Resiliencia (Schneider, 2007). De la misma manera que los empresarios aprenden a innovar y a establecer modelos de sostenibilidad, deben estar conscientes de los factores que les permiten ser resilientes (Piedrahíta Seifert, 2017).

Quiñonez \& Prado-Solis (2017) y XIAO \& CAO (2017) concluyen que la resiliencia organizacional es la capacidad de una organización para adaptarse en un entorno cambiante, para que pueda cumplir sus objetivos y para sobrevivir y prosperar. Se ha convertido en una competencia clave, por esto es importante promoverla, reflexionarla a través de la ética, la autoestima, la autorregulación emocional y otros aspectos (Rovayo Gabriel, 2013). Los altos ejecutivos de las organizaciones deben saber utilizar la resiliencia como la estrategia clave para poder competir, transformarse, y mantenerse a la vanguardia, saber enfrentar los problemas, para poder sobrevivir, desarrollarse y ser cada día más rentables.

\section{Conceptualización de Resiliencia}

El concepto de resiliencia no es un término recién conocido, ya que data de la década de los setenta en donde se empezaron a ver los primeros estudios sobre el temperamento de las personas, por investigadores estadounidenses. Además, por el deseo de conocer cómo las personas enfrentan la vida a distintas situaciones o momentos de transición y cómo estas personas crecen en condiciones adversas. A este grupo, se le denominó los resilientes. Rutter en 1979, expresaba que es importante conocer las razones por las cuales los individuos no resultan dañados, él hacía énfasis en que no se debe a factores protectores sino a mecanismos como fortalezas o aspectos positivos que los seres humanos usan para salir de situaciones de riesgo (Kotliarenco \& Fontecilla, 1997). En algunos casos, estas situaciones analizadas, eran de índole social, como la marginación, desempleo, desmotivación laboral, exposición y pertenencia a culturas de evasión y trasgresión, etc. (Flannery Diego, 2007).

Emily Werner en el año 1992, concluyó su trabajo investigativo por 32 años en el que se evaluaba a cada persona desde su nacimiento hasta la edad adulta, en los casos, cuyas familias habían sufrido 
penurias, pobreza extrema, violencia, peleas, alcoholismo, enfermedades con trastornos mentales, etc., de los 698 evaluados, 72 evolucionaron positivamente, es decir, el $10.32 \%$ de la muestra no necesitaron de algún tipo de tratamiento terapéutico, a esto se le denominó Resiliencia Humana, realizando un símil de la definición en la física, metalúrgica e ingeniería.(Flannery Diego, 2007). Como indicaba Werner y Smith en 1992, el pasar frente a una situación difícil, permite que el ser humano evolucione, atrayendo a su vez, nuevas vulnerabilidades y apoyos al cambiar los momentos vitales (Atehortíua Miguel, 2002).

La resiliencia ha provocado un cambio de paradigma en las disciplinas sociales y organizacionales, al hacer que este concepto permita la prevención, exploración, profundización y análisis de los problemas, fortaleciendo las capacidades de los individuos, grupos y sistemas formales (Villalva Quesada, 2003). El logro de alcanzar la Resiliencia permite acumular experiencia, trabajar en un pensamiento estratégico constante, con estrategias claras, llevando a las organizaciones a obtener un crecimiento y sostenibilidad.

En el ámbito empresarial, tanto ejecutivos como colaboradores estarán expuestos a momentos de presión, estrés y en ciertas circunstancias a tensión, que afectarán su desempeño profesional y equilibrio emocional, sin embargo y comúnmente, encontrarán una forma para seguir adelante. Es así, como se detalla a continuación el origen del vocablo resiliencia y algunas definiciones que serán de aporte a este documento.

La palabra resiliencia viene del latín resilire, que quiere decir volver atrás, volver a la posición original y rebotar. Este concepto que proviene de la física, se hace referencia a la capacidad de un material o de un sistema para volver al estado de equilibrio después de una deformación (Oviedo Amaro \& Marrero Santos, 2020). Luego de esta conceptualización, la resiliencia fue relacionada con las ciencias sociales y es así como en la psicología, esta es un proceso dinámico que abarca la adaptación positiva en entornos adversos (Becoña, 2006), teniendo como propiedades: la robustez, solidez, repetición, ingenio, iniciativa y rapidez (Bruneau \& Reinhorn, 2006).

De tal manera, que este concepto de enfrentar el riesgo permite al hombre o mujer, salir reforzado en cuanto a su nivel de madurez y desarrollo, ya que es una forma de prevención buscando una luz en medio de las sombras (Garrido \& Sotelo 2005). Uno de los pioneros en dar fuerza a este término, fue el investigador del BICE de Ginebra, Stephan Vanistendael, quien conceptualizó en el año 1994 a la Resiliencia como la capacidad de las personas para protegerse frente a adversidades y a su vez, a la capacidad de lograr una actitud positiva frente a estas complicadas circunstancias (Vanistendael, 2009).

Figura 1. Aspectos de la Resiliencia

Tomado de: Como salir del pozo (La Resiliencia) (Callabed, 2011). 
En la Figura 1, se presenta la gráfica de la "casita" del investigador Stephan Vanistendael (1994), que es la utilización de la metáfora en una ilustración sobre los aspectos de la resiliencia. Su objetivo es ayudar a construir, mantener y restablecer la misma. Toda persona que decida gestionar deberá saber usar las acciones precisas que pondrá en cada lugar de la casita. Brevemente se muestra, en el primer piso: El sentido de la vida, la proyección al futuro, la planificación de objetivos concretos. En el segundo nivel, la autoestima, las aptitudes personales y sociales, y finalmente, el sentido del humor. Cuando encontramos a estas competencias sociales y personales, de manera conjunta, la resiliencia relaciona aspectos como: el éxito social, la autonomía, las perspectivas de un futuro mejor, el optimismo, aptitudes relacionales y la habilidad para resolver problemas, es decir, la capacidad para elaborar estrategias, creatividad y un espíritu crítico. La resiliencia es un proceso coherente, que integra conocimientos y habilidades con una mirada más positiva sobre los seres humanos y la supervivencia empresarial (Muñoz Garrido \& De Pedro Sotelo, 2005).

\section{Resiliencia Organizacional}

Esta se define como aquellas empresas que tienen la capacidad de enfrentar cambios, internos y externos, a través de su gran flexibilidad de adaptación. Estas logran tener mayor estabilidad, y beneficios. (Minolli, 2000). En la teoría de la administración una organización resiliente hace frente a toda problemática, y en donde su habilidad para reinventarse de manera rápida, la caracteriza primordialmente (Gonzalez\&Castellano\& Piza, 2010). A su vez, estas organizaciones tienen bien definida su estrategia y saben transmitir a su staff, su sistema de valores y cultura, inculcando formas de comportamiento, basados en la confianza, trabajo en equipo, cooperación y transparencia. Estas serán mucho más exitosas al poder maximizar en estos términos el mayor potencial entre sus colaboradores y en cada departamento de la organización (Atehortíua Miguel, 2002).

Dentro de la perspectiva interna de la organización, los recursos humanos son uno de los que la caracterizan, al igual que la empatía y la habilidad social son marcos fundamentales para una abierta y buena comunicación, haciéndolas realmente resilientes (Minolli, 2000). En algunas situaciones, las empresas desarrollan mecanismos protectores para resilir frente a la crisis como, por ejemplo: el aprendizaje organizacional, es decir, el conocimiento interno, liderazgo estratégico, el clima laboral, la cultura y el cambio (Vega, 2012). Estos mecanismos se representan en la mandala de la resiliencia organizacional (ver Figura 2), misma que es necesaria para formar un todo, en busca de la unificación del individuo, de los departamentos y de la integración de toda la operación (Salvá Andria, 2011)

Figura 2. Mandala de la Resiliencia

Tomado de: El Mandala Organizacional. Reto de los Recursos Humanos (Salvá Andria, 2011).

Las normas ISO (International Organization for Standardization) identifican a la resiliencia organizacional como la habilidad para absorber y adaptarse en un entorno en constante cambio para cumplir sus objetivos y prosperar. 
ISO 34101-1:2019 capacidad de las personas u organizaciones para anticiparse, prepararse para responder, adaptarse y recuperarse ante los cambios, tensiones y perturbaciones

ISO 14080:2018 capacidad de los sistemas sociales, económicos y ambientales para hacer frente a un evento, tendencia o alteración peligrosos, respondiendo o reorganizándose de manera que se mantengan su función, identidad y estructura esenciales y al mismo tiempo su capacidad de adaptación, aprendizaje y transformación.

\section{MATERIALES Y MÉTODOS}

En la presente investigación se realizó una revisión documental en base de artículos científicos Latindex y Scielo, normas internacionales de gestión ISO, trabajo de tesis de maestría y estudio doctoral con el fin de analizar la aplicación del término resiliencia al contexto empresarial y los beneficios de desarrollar esta competencia en los administradores de empresas.

\section{RESULTADOS Y DISCUSIÓN}

Existen varios beneficios que tiene una organización resiliente, tales como: ventaja competitiva, la flexibilidad en su accionar, la adaptabilidad y al establecimiento de estrategias de comunicación con su staff, que romperán las barreras y antiguos paradigmas jerárquicos, que existían entre los mandos gerenciales, medios y bajos (Minolli, 2000). Las empresas exitosas, usarán la fuerza de sus equipos adaptables y comunicativos, mas no de la omnisciencia de su jerarquía (Vega, 2012).

Otro análisis importante que se debe indicar es la actitud clave que los Directivos y ejecutivos deben tener. Ellos deben considerar, invertir apropiadamente en proyectos nuevos y dejar el miedo al riesgo, salirse de la rutina y dejar de mirar hacia el pasado. Como lo indicaba Hammel y Valikangas en el 2003 "El riesgo es una función de la incertidumbre". En las organizaciones saludables, los ejecutivos son piezas relevantes y como lo transcribe Gabriel Rovallo, Director General de la escuela de negocios IDE, del Ecuador "El ejecutivo de hoy es global, conectado e innovador, resiliente y mucho más preparado, es espejo de un buen líder que genera confianza e invita a la reinvención constante". A pesar de lo recalcado anteriormente, el riesgo al fracaso existe, sin embargo, los ejecutivos con ambición, visionarios, que se atreven a aceptar proyectos de transformación, sin temores, descubrirán que éstos ofrecen oportunidades para su propio crecimiento personal y éxito profesional.

Además, algunas instituciones que les interesa estar inmersos en el éxito y la salud organizacional, desarrollan varios modelos que les permiten lograr este objetivo, como por ejemplo: El modelo HERO (Heakthy y Resilient Organizations), en donde Salanova y Schaufeli en el 2009, especificaba que si los factores claves como las prácticas de organización saludable, capital psicológico positivo y resultados saludables, se desarrollan de manera sistemática e interactiva, estos influirían en los procesos del trabajo en mejora constante en el tiempo. Otro modelo, es el de RED (Recursos, Emociones/Experiencias y Demandas), en donde el trabajo requiere de esfuerzo físico y psicológico, por ende, tendrá costos mentales y emocionales. Aquí se consideran las demandas laborales que causan consecuencias negativas, malestares para la organización y tiene repercusiones en las demandas laborales (Meneghel, 2009)

Es importante recalcar que la Norma ISO 22301:2019 de Seguridad y Resiliencia, precisa los requerimientos necesarios para un sistema de gestión de continuidad de negocios para poder responder y recuperarse ante cualquier riesgo o eventualidades que genere una interrupción de las operaciones. La resiliencia organizacional considera los siguientes beneficios (SP Consulting International Pte Ltd, 2020):

- Comportamiento alineado con la visión, misión y valores.

- Comunicación para fomentar el conocimiento compartido y el aprendizaje 
- Absorber, adaptarse y responder efectivamente al cambio.

- Proactividad y control del riesgo

- Diversidad de habilidades, liderazgo, conocimiento y experiencia

- Crea ventaja competitiva

Brevemente se puede indicar algunas herramientas que los Ceos o líderes deben tomar en cuenta, al momento de querer prevenir a largo plazo crisis organizacionales. Como por ejemplo existe el modelo de Virtudes de Competencia y Salud para el Trabajo (VICOSAT) que es usada por el trabajador para lograr un alto nivel de competencia, bienestar y salud laboral. Éste se basa en las teorías de la psicología positiva que estudia los cimientos de la felicidad y bienestar, las fortalezas, virtudes humanas (Antonio Sánchez, 2011). La denominada salud positiva que al ser aplicada en la persona alcanza un cambio transformador. (Vásquez\&Hervas, 2009). Para Goncalves Oliveira (2019) la resiliencia individual se asocia significativamente con indicadores de salud laboral (pág. 65).

Estas virtudes se han clasificado en fortalezas primarias (Optimismo, Auto eficacia Resiliencia) que repercuten directamente en la persona que las siente, las fortalezas secundarias (Honestidad, Lealtad y vinculación) que indicen en la persona que las rodea y finalmente las de mayor profundidad espiritual, que son las que tienen que ver con algún nivel de madurez emocional para transmitirlas, transcienden en el individuo y en su ambiente laboral, como gratitud, sabiduría y esperanza (González, 2010).

Según Kantur \& Iseri-Say (2015) para evaluar la percepción sobre resiliencia se evalúan 3 dimensiones: 1) robustez, que mide la capacidad de las organizaciones para resistir y recuperarse de condiciones desfavorables; 2) agilidad, que mide la capacidad de las organizaciones para tomar acciones rápidamente; e 3) integridad, que mide la cohesión de los empleados de la organización ante circunstancias desfavorables.

\section{CONCLUSIÓN}

La revisión de la literatura en relación el término resiliencia concluye que es la capacidad para analizar disrupciones, adaptarse a circunstancias cambiantes y recuperarse ante la adversidad. Las herramientas, habilidades y estrategias deben, sin lugar a duda, ayudar a que los colaboradores resilientes, tengan la capacidad de seguir adelante frente a las adversidades y evitar los efectos o resultados negativos como el fracaso o crisis. En el marco de la competitividad empresarial, la resiliencia es una competencia clave para los ejecutivos, debido que pueden establecer e implementar estrategias decisivas de prevención y aprendizaje, que le permitirán contar con equipo de trabajo mucho más comprometido con la empresa y reforzar el clima laboral.

Los cambios del entorno local demanda mayor competitividad a las organizaciones y formar administradores que tengan la capacidad de reaccionar al cambio de entorno y convertir los riesgos en oportunidades. Ante la crisis del covid-19, el análisis tradicional de amenazas y oportunidades escaló a convertirse en un plan de recuperación y de continuidad del negocio tomando mayor fuerza la parte interna organizacional conductual de individuos y grupos laborales.

Conjuntamente al análisis del comportamiento y virtudes de sus colaboradores, las organizaciones requieren fomentar una cultura de mejorar continua, liderazgo, comunicación eficiente, gestión del conocimiento y orientación a los objetivos, otorgando un ambiente agradable, productivo para así alcanzar una mayor rentabilidad en sus gestiones. Fomentando la resiliencia organizacional, se enfatiza el análisis de factores de riesgos, se conseguirá identificar amenazas, oportunidades y capacidad de recuperación ante eventos externos tanto económicos como sociales. 


\section{Futuras líneas de investigación}

Para Cristina Ruiz-Martin, Adolfo López-Paredes \& Gabriel Wainer (2018) es necesario medir las pérdidas económicas y los tiempos de recuperación ante eventos disruptivos. Por otra parte, para Sedano-Chiroque, Rojas-Miliano, \& Vela-Ruiz (2020) existe la necesidad de realizar valoraciones a la política de salud pública y preventiva del covid-19 basadas en evidencia científica, social y económico sobre todo en el contexto local del país. En este sentido, es importante evaluar casos de estudios como resultado de la crisis por la pandemia covid-19.

Otras investigaciones para desarrollar se deben relacionar con la gestión de crisis y la cultura de resiliencia organizacional. En consecuencia, es transcendental comunicar las lecciones aprendidas y analizar los aspectos vulnerables de diversos sectores económicos y que el conocimiento adquirido permita identificar criterios de decisión a ser aplicados en la planificación estratégica de las empresas.

Al analizar las necesidades del contexto laboral y social, adquiere relevancia desarrollar en los profesionales la competencia de resiliencia. Las universidades se interesan en garantizar que sus estudiantes adquieran los conocimientos y las habilidades que se requieren ante la globalización y la sociedad del conocimiento. En consecuencia, se sugiere evaluar los mecanismos orientados a incorporar la resiliencia en la formación de administradores de empresas y en la malla de estudios de carreras afines a la administración.

\section{REFERENCIAS}

Antonio Sanchez. (2011). Familia, trabajo y fiesta: un análisis desde la perspectiva de la Psicologia Positiva.From

http://www.upcomillas.es/redif/revista/volumen4/Famillia,\%20trabajo\%20y\%20fiesta.\%20S alamanca.pdf

Atehortíua Miguel. (2002). Resiliencia: otra perspectiva de las experiencias sociales y personales dentro de la empresa. (1. 4.-5. Estudios Gerenciales, Ed.) Obtenido de http://www.icesi.edu.co/revistas/index.php/estudios_gerenciales/article/view/72

Becoña, E. (.-1. (2006). Becoña, E. (2006). Resiliencia: definición, características y utilidad del concepto. Revista de psicopatología y psicología clínica, 11(3), 125-146.

Bruneau \& Reinhorn. (2006). Overview of the resilience concept. In Proceedings of the 8th US National Conference on Earthquake Engineering (pp. 18-22). Obtenido de http://wwwcivil.eng.buffalo.edu/ bruneau/8NCEE-Bruneau\%20Reinhorn\%20Resilience.pdf

Callabed, J. (2011). Como salir del pozo (La Resiliencia). Obtenido de http://www.andalan.es/?p=4996

Flannery Diego. (2007). Universidad Kennedy de Argentina. Obtenido de Universidad Kennedy de Argentina.:

http://www.kennedy.edu.ar/DocsDep18/Psicolog\%C3\%ADa\%20Preventiva/Resiliencia\%20 -\%20Otra\%20vida\%20es\%20posible.pdf

Gallopin, G. (2013). Sostenibilida y desarollo sostenible. Enfoque sistemico. Obtenido de http://www.eclac.org/publicaciones/xml/6/14256/lc11864p.pdf

Garrido \& Sotelo 2005. (s.f.). Revista complutense de educación. Educar para la resiliencia. Un cambio de mirada en la prevención de situaciones de riesgo social. Obtenido de Revista complutense de educación, 16(1), 107-124.: http://scholar.google.es/scholar?q=Educar+para+la+resiliencia.+Un+cambio+de+mirada+en +la+prevenci\%C3\%B3n+de+situaciones+de+riesgo+social+Victoria+MU\%C3\%91OZ+GA RRIDO+Universidad+Complutense+de+Madrid+Email\%3A+vmunozga\%40edu.ucm.es+Fr ancisco+DE+PEDRO+SOTELO

Goncalves Oliveira, L. (2019). Resiliencia en organizaciones de salud (tesis doctoral). Universitat Autónoma de Barcelona. 
Gonzalez\&Castellano\& Piza. (2010). Analisis y reflexiones

universitarias. La resiliencia organizacional. Obtenido de Analisis y reflexiones universitarias. La resiliencia organizacional. $\mathrm{P}, 107$ : http://incubadora.itson.mx/publicaciones/Documents/rada/analisisyreflexiones.pdf

González, C. (2010). Modelo integral de virtudes para competencia y salud trabajo. Obtenido de Universidad de las Americas, ciudad de méxico.: http://admin.udla.mx/mqr/index.php/mqr/article/view/59/28

Hamel, G., \& Välikangas, L. (2003). En busca de la resiliencia. Harvard Business Review, 81(9), 3339.

Obtenido http://www.planning.com.co/bd/archivos/1003_En_busca_de_la_resilencia_Gary_Hamel_Li isa_Valikangas.pdf

Kantur, D., \& Isery-Say, A. (2015). Measuring organizational resilience: A escale development. Journal of Business, Economics \& Finance, 4(3), 456-472. doi:https://doi.org/10.17261/Pressacademia.2015313066

Kotliarenco \& Fontecilla. (1997). Estado de arte en resiliencia. Obtenido de Washington DC: Organización Panamericana de la Salud.: https://www.uai.edu.ar/transferenciauniversitaria/aprendizaje-yservicio/ESTADO\%20DE\%20ARTE\%20EN\%20RESILIENCIA\%20$\% 20$ Organizacion $\% 20$ Panamericana\%20de\%20la\%20Salud\%20\%20Organizacion\%20Mundial\%20de\%20la\%20Salud.pdf

Maria Jose Fraile, D. d. (2009). Equipos y Talento Humano. Obtenido de http://www.equiposytalento.com:

http://www.equiposytalento.com/tribunas/meta4/fomentando-la-resiliencia

Meneghel, I. (2009). La Resilencia en el Desarrollo de la Organización Saludable.Proyecto de Investigación. Universitat Jaume. From Proyecto de Investigación: https://www.researchgate.net/publication/262033860_La_resiliencia_en_el_desarrollo_de_1 a_organizacion_saludable

Minolli, C. (2000). Empresas resiliente. Algunas ideas para construirlas. Obtenido de http://www.ucema.edu.ar/u/cpcbm/Investigacion/Resiliencia/Empresas_Resilientes.pdf

Muñoz Garrido, V., \& De Pedro Sotelo, F. (2005). Educar para la resiliencia. Un cambio de mirada en la prevención de situaciones de riesgo social. Revista Complutense de Educación, 16(1), $107-124$.

Obtenido

de https://revistas.ucm.es/index.php/RCED/article/view/RCED0505120107A

Oviedo Amaro, Y., \& Marrero Santos, M. d. (2020). La resiliencia organizacional. Un constructo para empoderar a las empresas. Revista Cubana de Salud y Turismo, 21(2), 66-72. Obtenido de http://www.revsaludtrabajo.sld.cu/index.php/revsyt/article/view/144

Piedrahíta Seifert, T. (2017). Organizational Resilience: Key Factors to overcome and survive a Business Crisis. Learnings from a family owned business in Crisis. (Tesis de maestria). Malmo University, Faculty of Culture and Society. Obtenido de http://www.divaportal.org/smash/record.jsf?pid=diva2\%3A1481286\&dswid=2049

Quiñonez, R., \& Prado-Solis, M. (2017). Resiliencia organizacional: ideas para el debate en el contexto ecuatoriano. Dominio de las Ciencias, 7(6), 488-504. Obtenido de https://dominiodelasciencias.com/ojs/index.php/es/index

Revista Ekos Investigacion. (2011). Ekos Negocios. Obtenido de http://www.ekosnegocios.com/negocios/especiales/documentos/empresasMasRentables.pdf

Rovayo Gabriel, D. I. (Febrero de 2013). Revista Perspectiva. Obtenido de http://investiga.ide.edu.ec/index.php/revista-perspectiva/184-febrero-2013/935-resilienciauna-competencia-indispensable-de-todo-buen-gerente

Ruiz-Martin, C., López-Paredes, A., \& Wainer, G. (2018). What we know and do not know about organizational resilience. International Journal of Production Management and Engineering, 6(1). doi:https://doi.org/10.4995/ijpme.2018.7898 
Salvá Andria. (Julio de 2011). EL Mandala organizacional. Reto de los Recursos Humanos. Obtenido de Andria M. Salvá, Ph.D. Presidente EU Quest Talent Consulting: http://www.zonaderecursos.com/articulos/item/el-mandala-organizacional-retos-derecursos-humanos

Schneider, B. (2007). Resiliencia: Como construir en contextos de inestabilidad. Editorial Norma, 2007. Bogotá, Colombia. Obtenido de http://books.google.es/books?hl=es\&lr=\&id=zDE91OQroEC\&oi=fnd \&pg=PA15\&dq=Schneider,$+B+(2007) .+$ Resiliencia:+Como+construir + en +contextos+de+inestabilidad.+Editorial+Norma,+2007.+Bogot\%C3\%A1,+Colombia.\&ots= Rrb6cq6Sml\&sig=CyTiPTNANygaX-EPeGatzMB-eFc\#v=onepage

Sedano-Chiroque, F. L., Rojas-Miliano, C., \& Vela-Ruiz, J. M. (Julio - Septiembre de 2020). COVID-19 desde la perspectiva de la prevención primaria. Revista de la Facultad de Medicina Humana, 20(3), 494-501. doi:http://dx.doi.org/10.25176/rfmh.v20i3.3031

SP Consulting (International) Pte Ltd. (2020). Security and resilience - Business continuity management systems - Requirements. Singapore Standards. Enterprise Singapore.

Vanistendael, S. (2009). La resiliencia o el niño del 'diente de león'. Monitor educador, (131), 6-7.

Vasquez\&Hervas. (2009). Psicologia Positiva Aplicada 2da Edicion. (D. d. Bourwer, Ed.) From http://bibliotecadigitalhispanica.bne.es/webclient/DeliveryManager?pid=1812788

Vega, R. (2012). Ventaja competitiva en una organizacion resiliente (Trabajo de grado). Colegio Mayor de Nuestra Señora del Rosario.

Obtenido de http://repository.urosario.edu.co/bitstream/10336/2879/1/1018423819-2012.pdf

Villalva Quesada, C. (2003). El concepto de resiliencia individual y familiar. Aplicaciones en la intervención social. Psychosocial Intervention, 12(3), 283-299. Obtenido de https://journals.copmadrid.org/pi/art/931af583573227f0220bc568c65ce104

XIAO, L., \& CAO, H. (2017). Organizational Resilience: The Theoretical Model and Research Implication. ITM Web of Conferences 12. doi:DOI: 10.1051/ 71204021

\section{CONFLICTOS DE INTERESES}

Los autores no refieren conflictos de intereses 